Katharina Heyden und Martin Wallraff*

\title{
Centro Melantone. Ökumenisches Leben und Lernen in Rom
}

DOI 10.1515/zpt-2016-0032

Abstract: The Melanchthon Center in Rome was founded by Waldensians and Lutherans at the turn of this millennium and offers Protestant theologians a place for study and research from an ecumenical point of view. This article sets the project in its historical and ecclesiastical context and aims for the first time to write its history in a way that includes the perspective and experiences of the participants themselves. After a description of the center's primary activities (the program of studies, courses, events and publications), we consider the possibilities for theological education in a transnational and transdenominational setting, taking stock of the past and looking ahead at the prospects for developments in the future.

Zusammenfassung: Das Melanchthon-Zentrum in Rom wurde von Waldensern und Lutheranern um die Jahrtausendwende gegründet. Es bietet einen Studienund Forschungsraum für protestantische Theolog/innen in ökumenischer Perspektive. Der Artikel ordnet die Initiative in ihren historischen und kirchlichen Kontext ein und versucht zum ersten Mal, ihre Geschichte zu schreiben - teilweise aus der Perspektive der direkt Beteiligten. Nach einer Beschreibung der konkreten Aktivitäten (Studienjahr, Kurse, Tagungen, Publikationen) lotet er das Potenzial für die transnationale und transkonfessionelle theologische Bildung aus. Dies geschieht reflexiv und prospektiv: im Nachdenken über das Zurückliegende und im Ausblick auf mögliche Entwicklungsperspektiven.

Keywords: Rome, Ecumenism, Theological education

Schlagworte: Rom, Ökumene, theologische Bildung

*Kontakt: Prof. Dr. Katharina Heyden, Theologische Fakultät, Universität Bern, Länggassstraße 51, 3012 Bern, CH, E-Mail: katharina.heyden@theol.unibe.ch

Prof. Dr. Martin Wallraff, Evangelisch-theologische Fakultät, Ludwig-Maximilians-Universität München, Geschwister-Scholl-Platz 1, 80539 München, De, E-Mail: Martin.Wallraff@lmu.de 


\section{Rom als Bildungsort für protestantische Theologie}

Die Stadt Rom ist für viele europäische Protestanten seit der Reformationszeit prägend gewesen - als konkreter wie auch als metaphorischer Ort, als Inspirationsquelle oder als Inbegriff konfessioneller Polemik. ${ }^{1}$ Letzteres überwog natürlich zunächst, doch geht auch die Faszination für Rom tiefer und weiter zurück als die ökumenische Bewegung des 20. Jahrhunderts. Während in Kunst, Literatur und Wissenschaft neben individuellen Einzelerfahrungen seit längerem auch institutionelle Kanäle vorhanden sind (man denke nur an die ausländischen Kulturinstitute in Rom, für deren Gründung und Geschichte teilweise Protestanten eine wichtige Rolle spielten), war Rom als theologischer Bildungsort bis vor verhältnismäßig kurzem nur von nationaler Bedeutung: Die italienischen Reformierten und Methodisten bilden seit Mitte des 19. Jahrhunderts ihren Nachwuchs an der Waldenserfakultät in Rom aus.

Seit gut anderthalb Jahrzehnten bietet das Melanchthon-Zentrum für ökumenische Studien in Rom / Centro Filippo Melantone. Centro protestante di studi ecumenici nun auf breiterer Basis evangelischen Theologinnen und Theologen vielfältige Möglichkeiten, sich in der Hauptstadt des Katholizismus mit ökumenischen Studien, mit Leben und Lehre der römisch-katholischen Konfession und mit waldensischer Theologie und Identität in der Diaspora zu befassen. Als gemeinsame Initiative der Waldenserfakultät und der Evangelisch-Lutherischen Kirche in Italien (ELKI) ist die Basis formal weiterhin national, doch schon Geschichte und Profil der ELKI, die zu guten Teilen im deutschsprachigen Protestantismus wurzelt, macht deutlich, dass der Horizont weiter ist. Das Centro Melantone versteht sich ,als Plattform der Forschung und des Dialogs im Bereich der europäischen Kirchen, die die Leuenberger Konkordie unterschrieben haben“" (so bereits das Gründungsdokument von 2002), insofern also als ein transnationales Projekt. Der Bezug auf die „Gemeinschaft Evangelischer Kirchen in Europa (GEKE)“ ist konstitutiv, im speziellen hat die Achse zwischen Deutschland und Italien von Anfang an eine wichtige Rolle gespielt (institutionell vertreten durch die EKD). Neben dem transnationalen ist aber auch der transkonfessionelle Aspekt wichtig, und zwar

1 Für diverse Beispiele vom späten 18. bis ins frühe 20. Jahrhundert siehe die Beiträge in dem Band: Rombilder im deutschsprachigen Protestantismus. Begegnungen mit der Stadt im ,langen 19. Jahrhundert“, hg. v. Martin Wallraff, Michael Matheus und Jörg Lauster unter Mitarbeit von Florian Wöller. Tübingen (Mohr Siebeck) 2011. Dieses Buch ist der erste Band der Reihe „Rom und Protestantismus. Schriften des Melanchthon-Zentrums in Rom“. 
zum einen im Blick auf Protestanten und Katholiken, zum anderen aber auch in Bezug auf die innerprotestantische Ökumene.

Das Anliegen und die Geschichte der Aktivitäten des Centro Melantone werden hier erstmals in wissenschaftlichem Kontext dargestellt und reflektiert. Dass beide Autoren dieses Beitrags in enger Verbindung zum behandelten Gegenstand stehen - der eine als Mitbegründer des Zentrums, Mitglied im Wissenschaftlichen Beirat, Leiter zahlreicher Sommerkurse und Zweiter Vorsitzender des Freundeskreises, die andere als Teilnehmerin des ersten Studienjahrs, Sommerkursleiterin und Erste Vorsitzende des Freundeskreises -, kommt dem Beitrag einerseits zugute, setzt ihm aber im Blick auf eine vielleicht wünschenswerte kritische Distanz zum Thema zugleich Grenzen. Dies umso mehr, als beide Autoren das Fach Kirchengeschichte vertreten und Fremdlinge auf dem Gebiet der Bildungsforschung sind. Dennoch soll der Versuch unternommen werden, nach der Darstellung der (Vor-) Geschichte des Centro Melantone (2.) einige Überlegungen zu Anknüpfungspunkten an die im Einführungsartikel von David Käbisch skizzierten Fragen der aktuellen transnationalen Bildungsforschung zu formulieren (3.).

\section{2. (Vor-)Geschichte des Centro Melantone}

Wie alt ist das Centro Melantone? Man kann auf diese einfache Frage unterschiedliche Antworten geben, denn vor seiner Geschichte hat das Centro eine längere Vorgeschichte, mit anderen Worten: es gibt ein Centro Melantone ante litteram. Die Gründung ist erst im dritten Anlauf geglückt. Wenn man nicht gar in die 1920er Jahre zurückgehen möchte (damals hat Pfr. Ernst Schubert an der deutschsprachigen Gemeinde die Idee eines „Auslandsseminars“ in Rom ventiliert ${ }^{2}$ ), bietet sich als Einsatzpunkt die Zeit des Zweiten Vatikanischen Konzils an. In der ökumenischen Euphorie der damaligen Zeit ist Rom als Standort zum theologischen Studium auch für den Protestantismus in den Blick geraten. Offenbar waren es die Konzilsbeobachter von außerhalb Italiens, die hier einen ersten Impuls gaben. Dieser wurde dann auch von der Waldenserfakultät aufgenommen und weiterverfolgt - freilich mit charakteristischen Unterschieden. Während etwa Oscar Cullmann in einem Brief an Dekan Valdo Vinay vom 16.1.1966 von einem „ökumenischen Institut“ spricht, hatte die Fakultät wenige Tage zuvor (an ihrer Sitzung vom 12.1.1966) ausführlich über die Gründung eines „Istituto per

2 Siehe dazu im Evangelischen Zentralarchiv in Berlin die Verzeichniseinheiten 5/1557 und 5/1550. Für diesen Hinweis danken wir Frau Julia Früh (Tübingen). 
lo studio del cattolicesimo (Institut zum Studium des Katholizismus)“ debattiert ${ }^{3}$. Dieser Prozess entwickelte in den folgenden Monaten eine gewisse Dynamik (bis hin zu einer langen Beratung gemeinsam mit der waldensischen Kirchenleitung [tavola] am 22.8.1966), wobei freilich das Projekt den Skeptikern überhaupt nur durch den Hinweis schmackhaft gemacht werden konnte, es solle über und nicht mit dem Katholizismus gesprochen werden.

Obwohl die Korrespondenz in dieser Sache weite Kreise zog und obwohl die internationalen Gesprächspartner (etwa aus Deutschland, der Schweiz oder den USA) überwiegend ermutigende Signale gaben, ist das Projekt damals nicht in Gang gekommen. Es hielt sich dennoch in der memoria der Fakultät, namentlich in derjenigen des jungen Pfarrers, späteren Professors Paolo Ricca. Als dieser in den 80er Jahren das Amt des Dekans bekleidete, kam es nochmals zu einem Anlauf, der jedoch wiederum nicht den gewünschten Erfolg hatte.

Erst in den späten 90er Jahren kam Bewegung in die Initiative. Im Februar/März 1996 hielt der Tübinger Neutestamentler Martin Hengel Vorlesungen am Biblicum, dem päpstlichen Institut für biblische Exegese. Die dabei gewonnenen Eindrücke brachten ihn dazu, konkrete Schritte einzuleiten, um die Chancen des intellektuellen Austauschs und der historischen Ausbildung vor Ort auch protestantischen Theologinnen und Theologen zu ermöglichen ${ }^{4}$. Im Sommer 1996 involvierte er in ausgeweiteter Korrespondenz vor allem deutsche Gesprächspartner: den Ratsvorsitzenden der EKD Bischof Klaus Engelhardt, den Botschafter beim Heiligen Stuhl Philipp Jenninger, den Kurienkardinal (und späteren Papst) Joseph Ratzinger und andere. Das Projekt firmierte unter der Bezeichnung ,evangelisches Studienhaus in Rom“, und schon damals erschien zum ersten Mal die Immobilie auf dem Gelände des Deutschen Historischen Instituts am Horizont, die später tatsächlich gekauft und für diesen Zweck genutzt werden konnte.

Vor Ort waren vor allem der schon genannte Prof. Paolo Ricca und der Pfarrer der lutherischen Gemeinde Hans-Michael Uhl mit dem Projekt befasst. Ersterer schrieb im Mai 1997 ein Konzeptpapier („Linee per un progetto“); Letzterer trieb die Verhandlungen zur Verwendung der leerstehenden Immobilie voran. Sei es weil die waldensischen und lutherischen Bestrebungen nicht hinreichend koordiniert erschienen, sei es weil mehr von Strukturen als von konkreten Aktivitäten die Rede war - der Prozess drohte wiederum ins Stocken zu geraten. In merkwürdiger Gleichzeitigkeit teilte der Rat der EKD im Frühsommer 1999 mit, dass er

3 Dies und die folgende Informationen aus dem Archiv der Waldenserfakultät, insbesondere „Verbali del Consiglio 1960-73“ und „Corrispondenza Decano 1965-66“.

4 Das Folgende nach einem Aktenkonvolut von Pfr. Hans-Michael Uhl, das dem Verfasser in Kopie vorliegt. 
das Projekt nicht weiter verfolgen würde - und wurden vor Ort konkrete Aktivitäten geplant.

Der Verfasser der vorliegenden Zeilen stand vor dem Abschluss seiner Habilitation und plante, im Anschluss als Vikar nach Rom zu gehen, um dort gemeinsam mit Pfr. Uhl die Initiative durch konkrete Studienangebote zu stärken - in der Hoffnung, dass die reale Nachfrage das politische Interesse an dem Projekt förden würde. In diesem Sinne fand im September des Jubeljahres 2000 als eine Art Pilotprojekt ein 14-tägiger „Sommerkurs ökumenisches Lernen in Rom“ statt. Dieser stieß tatsächlich auf großes Interesse (etwa doppelt so viele Bewerbungen wie Plätze), so dass der Weg frei war für eine weitere Initiative: die Ausschreibung zu einem Studienjahr in Rom. Diese erfolgte im November 2000 - für das Studienjahr 2001/02. Auch für diese Initiative war das Interesse größer als zunächst gedacht. Die Unterbringung der Studierenden war - etwas provisorisch - mit Mitteln der Gemeinde und über ökumenische Kontakte möglich.

Damit ergab sich die eigentümliche Situation, dass das Projekt offiziell-institutionell gescheitert, aber faktisch funktionierend und lebendig war. Noch während des ersten Studienjahres wurden daher Schritte zur Gründung eines Studienzentrums gegangen - zunächst noch ganz ohne juristische Form (von eigenem Haushalt und Immobilie einmal gar nicht zu reden). In dieser Phase ging die Initiative von den lokalen stakeholders aus, also nicht von ausländischen Instanzen, etwa in der Weise eines deutschen „Auslandsinstituts“. Ein sehr knappes Gründungsdokument (kaum mehr als eine Absichtserklärung) wurde am 15.3.2002 von der Waldenserfakultät verabschiedet, dann am 29.4. von der Synode der evangelisch-lutherischen Kirche in Italien (ELKI) und schließlich am 28.8. von der Synode der Waldenserkirche. Die Namengebung war damals Gegenstand von Diskussionen. Neben Philipp Melanchthon (italienisch: Filippo Melantone) standen noch zwei Theologieprofessoren des 20. Jahrhunderts als „Patrone“ zur Debatte: Giovanni Miegge (1900-61, Neutestamentler und Luther-Forscher an der Waldenserfakultät) und Oscar Cullmann (1902-99, Neutestamentler und Ökumeniker an der Universität Basel mit vielfachen Beziehungen nach Rom). Melanchthon war in diesem Zusammenhang zum ersten Mal in einem Konzeptpapier der EKD vom September 1997 aufgetaucht („Philipp-Melanchthon-Studienhaus“), doch war die Entscheidung für den großen Humanisten unter den Reformatoren im Jahr 2002 davon unabhängig.

Die beiden schon genannten Aktivitäten - Sommerkurs und Studienjahr sollten regelmäßig fortgeführt werden; tatsächlich sind sie seither zum kontinuierlichen Kernbestand der Arbeit des Centro Melantone geworden. Vor allem für das Studienjahr war die Unterbringungsfrage eine konstante Sorge. Neben der Wohnung der lutherischen Gemeinde in Via Savoia (die auch nicht zur Gänze zur Verfügung stand) mussten jeweils zusätzliche Zimmer gesucht und gefunden 
werden. Dem ELKI-Dekan Jürgen Astfalk ist es zu verdanken, dass die Verhandlungen über die oben schon genannte Immobilie in Via Aurelia antica wieder aufgenommen wurden - und zwar jetzt mit dem Gedanken, neben dem Studienzentrum auch für das Dekanat der ELKI einen neuen Sitz zu finden. Die Entscheidung zum Ankauf der oberen beiden Stockwerke des seit Jahren leerstehenden Hauses durch die Kirche wurde 2004 getroffen. Drei weitere Jahre vergingen bis zum Abschluss der umfangreichen Sanierungsarbeiten (Eröffnung im September 2007). Seither könnten einige Studierende im Haus wohnen, andere wohnen im Konvikt der Waldenserfakultät und noch andere ziehen es vor, sich etwas auf dem freien Markt zu suchen.

Im gleichen Jahr 2007 gab sich das „Centro Filippo Melantone. Centro protestante di studi ecumenici“ (so unterdessen die offizielle Bezeichnung) ein Statut und wurde damit zur juristischen Person (Verein italienischen Rechtes). Die Grundstruktur entspricht dem Geist des Gründungsdokuments von 2002, nämlich die paritätische Verantwortung von Waldenserfakultät und lutherischer Kirche als Träger des Zentrums. Während zuvor die Leitung eher informell durch ein vierköpfiges Leitungsgremium (comitato direttivo) erfolgte, stand nun eine feste institutionelle Struktur zur Verfügung: ein Vorstand (etwas ungeschickt als „Leitungsrat“ aus dem italienischen „,consiglio direttivo“ übersetzt) mit einem Präsidenten, eine jährlich stattfindende Generalversammlung und die Möglichkeit zur Einberufung eines wissenschaftlichen Beirats. Erster Präsident wurde der ELKIDekan Holger Milkau. Seit 2013 hat Prof. Daniele Garrone von der Waldenserfakultät das Amt inne. Zudem enthält das Statut auch von vorne herein die Möglichkeit zur Assoziierung weiterer Partner. Als wichtigste internationale Begleiter sind EKD und GEKE bereits „fest verdrahtet“. Mit beiden wurden in der Folge auch förmliche Vereinbarungen geschlossen, um die Zusammenarbeit auf eine feste Grundlage zu stellen. Besonders die Unterstützung durch die EKD ist wichtig - nicht nur wegen eines, allerdings nicht allzu hohen, Unterstützungsbeitrags, sondern vor allem weil sie sich bereit erklärt hat, dem Studienleiter eine Wohnung in der EKD-eigenen Immobilie in Via Toscana 7 (lutherisches Gemeindehaus) zur Verfügung zu stellen.

Neben - und eigentlich vor - Fragen von Immobilien und Institutionen ist diese Figur der Schlüssel zum Erfolg des Zentrums. Seit Gründung haben sechs Personen diese verantwortungsvolle Aufgabe wahrgenommen. Studienleiter ante litteram waren der Schreiber dieser Zeilen sowie Vikar Dr. Jens-Martin Kruse. Es folgten Dr. Jochen Rexer (2003-06), Dr. Friedemann Steck (2006/07), Philine Blum (2008-10), (jetzt: Dr.) Michael Jonas (2010-12), Ulrike Eichler (2012/13) sowie Tobias Küenzlen (ab 2013). Mit der Ausnahme von Steck (Bayern) und Eichler (Westfalen) wurden diese Personen von der Württembergischen Landeskirche entsandt und vor allem auch finanziert. 
Während die ersten Studienjahre aus den genannten logistischen Gründen noch kleiner waren, hat sich die Teilnehmerzahl im zurückliegenden Jahrzehnt um die 10 eingependelt. Insgesamt haben (bis einschließlich Jahrgang 2015/16) 134 Theologiestudierende von der Möglichkeit Gebrauch gemacht, ein Jahr lang mit Unterstützung des Centro Melantone in Rom zu studieren. Die Sommerkurse haben üblicherweise Teilnehmerzahlen von 15-20, so dass seither weit über 200 Personen diese römische Erfahrung gemacht haben. (2013 fand kein Sommerkurs statt - dafür gab es im Jahr 2009 deren zwei.) Einige Kurse hatten ein spezifisches Thema („Interkulturelle Theologie“, „Kirchengeschichte Roms“, „Diaspora“, „Maria“), andere haben das ökumenische „Grundthema“ behandelt. Die Leitung war jeweils in der Hand eines deutschsprachigen Hochschullehrers mit Unterstützung durch den Studienleiter.

Von den weiteren Aktivitäten des Centro kann hier nur sehr summarisch gesprochen werden. Im Lauf der Zeit ist das Kursprogramm stark ausgeweitet worden. Mehrmals fanden Fortbildungskurse für Religionslehrer/innen sowie für Pfarrer/innen statt (beides in deutscher Sprache). Zweimal fanden „Winterkurse“ (als Pendant zum Sommerkurs) in englischer Sprache und mit einer primär amerikanischen Zielgruppe statt. Zukunftsweisend ist das Angebot von Kursen für Promovierende.

Noch stärker in den Bereich Wissenschaft und Forschung geht die Organisation von Tagungen. Mehrere internationale Initiativen haben stattgefunden (,Rombilder im deutschsprachigen Protestantismus“, „Katholizismus heute“, „Luther in Rom"), weitere sind in Vorbereitung. Die Resultate dieser Tagungen sowie andere themen-affine Manuskripte werden in der wissenschaftlichen Reihe des Centro Melantone publiziert („Rom und Protestantismus“ bei den Verlagen Mohr Siebeck und Claudiana) ${ }^{5}$.

Kaum im einzelnen aufzulisten, aber in der Praxis umso wichtiger, ist die Vortragstätigkeit (Einladung an auswärtige Referierende, eigene Vorträge des Studienleiters oder der Mitglieder des Vorstandes) sowie die ökumenische Vernetzung vor Ort (Kontakt mit päpstlichen Hochschulen, mit römisch-katholischen kirchlichen Stellen, mit dem italienischen Protestantismus). Vernetzung über den römischen Horizont hinaus geschieht in Rom gleichfalls sehr einfach und unkompliziert, denn die Welt kommt früher oder später hierhin. Zahlreiche akademische Exkursionen, Pfarrkonvente, kirchliche Delegationen etc. suchen den Kontakt mit dem Centro Melantone.

Last, but not least seien noch zwei Instanzen der Begleitung genannt: Am 11. Juni 2003 wurde in Deutschland ein „Freundeskreis“ (als eingetragener Verein)

5 Als nächstes wird erscheinen: „Monotheismus, ein ganz leeres Wort“? Versuche zur Monotheismustheorie Erik Petersons, hg. v. Giancarlo Caronello. Tübingen (Mohr Siebeck) 2016. 
gegründet. Dieser hat unterdessen ca. 100 Mitglieder - zumeist ehemalige „Melantonini“ oder Hochschullehrer, die der Initiative verbunden sind. Der letztgenannte Personenkreis bildet auch das „Reservoir“ für den wissenschaftlichen Beirat, der im Statut des Centro vorgesehen ist. Er wurde im Oktober 2009 konstituiert. Derzeit gehören ihm sechs Professor/innen aus drei Ländern an.

\section{Das Melanchthon-Zentrum als Bildungsraum: transnationale und transkonfessionelle Aspekte}

Im folgenden werden einige Aspekte der Arbeit des Melanchthon-Zentrums genannt, die im Blick auf die transnationale Bildungsforschung von Interesse sein könnten.

a) Komparatistik und Kulturtransfer: Die Zeit deutscher „Auslandsinstitute“ ist vorbei. In diesem Sinne ist das Centro Melantone kein „inter-nationales“ (nämlich: von einer Nation betriebenes, sich einer anderen zuwendendes) Projekt, sondern im besten Sinne „transnational“ (nämlich: nationale Paradigmen einbeziehend, sie zugleich aber auch transzendierend). Die Bildungserfahrungen, die das Zentrum ermöglicht, reichen weit über die Beziehung zweier Staaten hinaus. Da die theologische Ausbildung in Italien nicht von den staatlichen Universitäten, sondern von kirchlichen Hochschulen verantwortet wird, finden sich die Teilnehmer des Studienjahres in einer ungewohnt vielfältigen Bildungslandschaft wieder. Neben den päpstlichen Universitäten und Instituten (z. B. Gregoriana, Biblicum, Augustinianum, Lateran-Universität) unterhalten die großen Orden und Gemeinschaften (Benediktiner, Dominikaner, Franziskaner etc.) in Rom eigene Ausbildungsstätten. Die Studierenden kommen buchstäblich aus aller Herren Länder. Nicht selten trifft man auf Theologiestudierende aus Lateinamerika, Asien oder Afrika, die von den katholischen Bildungsinstituten Roms auf eine spezifisch abendländische theologische Tradition hin orientiert werden. Angesichts dieses von „Rom“ intendierten, aber nicht immer gelingenden Kulturtransfers der abendländischen Denktradition(en) in die verschiedenen Kulturen der Weltkirche hinein treten die in Deutschland wahrnehmbaren konfessionellen Unterschiede bisweilen in den Hintergrund: römischer Katholizismus und aus der Reformation hervorgegangene Traditionen, die im deutschen Raum häufig als sehr gegensätzlich wahrgenommen werden, können angesichts der Begegnung mit viel fremderen christlichen Kulturen als Erben derselben abendländischen Denktradition erkannt werden. Wiederum wird aber in Rom auch deutlich, dass gerade der deutsche Katholizismus eine zwar einflussreiche, aber längst nicht die prägende Größe in Lehre und Leben der katholischen Weltkirche ist. 
Hinzu kommt ein weiterer Aspekt: Die meisten katholischen Studierenden wohnen entweder direkt in den Ordenshäusern oder in einem der zahlreichen, von einzelnen Nationen getragenen Priesterseminaren Roms. Neben die universitäre theologische Ausbildung tritt somit eine (im Fall der Ordenshäuser) internationale oder (im Fall der Priesterseminare) nationale Prägung des Lebensstils. Die meisten „Melantonini“ machen eine ähnliche Erfahrung - jedenfalls, sofern sie entweder in der Wohngemeinschaft im Studienhaus oder im Waldenserkonvikt wohnen. Die enge Verbindung von Lehre und Lebensweise bildet einen wesentlichen Unterschied zur theologischen Ausbildung in Deutschland, die in erster Linie an staatlichen Universitäten stattfindet. In den theologischen Ausbildungsstätten Roms herrscht denn auch ein Bildungsverständnis, das Lehre und Leben explizit miteinander verbindet und deren Verhältnis reflektiert.

Kurz gesagt: Rom ist ein Ort, an dem sich die Kontextualität von Theologien in vielfältiger Weise beobachten, selbst erleben und reflektieren lässt. Dies ist in religiös und konfessionell pluralen Gesellschaften zweifellos eine zukunftsweisende Grundaufgabe theologischer Ausbildung.

b) Im Rahmen der Beziehungsgeschichte lassen sich Phänomene sowohl auf persönlicher wie auch auf institutioneller Ebene nennen. Zunächst, auf persönlicher Ebene, bewerben sich regelmäßig Teilnehmer/innen des Sommerkurses für die Aufnahme in das Studienjahr. Viele Absolventen kommen später als Vikare oder Pfarrerin mit ihren Gemeindegruppen nach Rom und profitieren von den ökumenischen Kontakten, die das Centro Melantone hält und vermittelt. Für andere ergaben sich aus den im Centro Melantone geknüpften Kontakten weitere Forschungsbeziehungen: So sind seit Bestehen des Centro mehrere Dissertationen ehemaliger Studienjährler abgeschlossen worden, zwei davon mit spezifisch stadtrömischen Themen. ${ }^{6}$ Zudem sind zwei der bisherigen Studienleiter selbst Absolventen des Studienjahres gewesen. Was darüber hinaus an persönlichen Freundschaften und Arbeitsbeziehungen in Sommerkursen und Studienjahren entstanden ist und welchen Einfluss diese Verbindungen auf die pfarramtliche und wissenschaftliche Arbeit der Rückkehrer haben, ist bisher nicht untersucht worden. Die dafür notwendige Generierung und Auswertung empirischer Daten (Interviews, Fragebögen, eine wissenschaftlichen Maßstäben genügende Analyse schriftlicher Studienberichte, Netzwerkanalyse, wie sie etwa die Alumni-Studien des DAAD leisten) ginge über die mit diesem Beitrag intendierte erste Bestandsaufnahme hinaus in das Feld der empirischen Bildungsforschung.

6 Thomas Krönung, Vom Privathaus zum locus sacer. Die Entwicklung der römischen Titelkirchen in der Spätantike (Jena 2008); András Handl, Callixtus I., der Bischof von Rom und der Konflikt um seine Person in der Refutatio omnium haeresium (Basel 2015). 
Auf institutioneller Ebene ist die Intensivierung der innerprotestantischen Ökumene (v. a. zwischen Waldensern und Lutheranern) in der Stadt Rom und darüber hinaus (etwa in der finanziellen Unterstützung der Aktivitäten durch die EKD, die GEKE und den LWB) eine Frucht der Arbeit des Centro Melantone. Es ist gewiss auch keine Übertreibung zu behaupten, dass die Sichtbarkeit des Protestantismus in der Stadt Rom auch in der Wahrnehmung von katholischen Personen und Institutionen mit der Arbeit des Centro Melantone zugenommen hat.

c) Was schließlich die im Rahmen der Verflechtungsgeschichte in den Blick genommene Frage nach Rückwirkungen von transnationaler bzw. transkonfessioneller Bildung auf die Ausgangskultur betrifft, so mögen wenige Worte genügen: Seit den ersten Anfängen war es ein Anliegen der Förderer des Centro Melantone, nicht nur deutschen, sondern evangelischen Theologinnen und Theologen auch aus anderen europäischen und außereuropäischen Ländern die Möglichkeit einer Beschäftigung mit dem römischen Katholizismus vor Ort zu geben. Dass dies in Einzelfällen bereits gelungen ist, bezeugen Teilnehmer/innen in Sommerkursen und Studienjahren aus der Schweiz, Österreich, Tschechien, der Slowakei, Ungarn, Polen, Holland und Dänemark. Der innerprotestantischen Vernetzung in Europa soll aber in Zukunft noch mehr Aufmerksamkeit gewidmet werden. Vielleicht kann man diesen Versuch, nationale Verengungen der protestantischen Theologie ausgerechnet in Rom und von Rom ausgehend zu durchbrechen und den Protestantismus auf diese Weise ein wenig „katholischer“ $\mathrm{zu}$ gestalten, als eine Rückwirkung der römischen Weltkirche auf die protestantische Konfessionskultur verstehen. 